\title{
A FRACTIONAL FRAMEWORK FOR PERIMETERS AND PHASE TRANSITIONS
}

\author{
ENRICO VALDINOCI
}

\begin{abstract}
We review some recent results on minimisers of a non-local perimeter functional, in connection with some phase coexistence models whose diffusion term is given by the fractional Laplacian.
\end{abstract}

\section{The Fractional Perimeter}

A notion of fractional perimeter was introduced in [8]. To introduce it in a soft way, we consider a (measurable) set $E \subseteq \mathbb{R}^{n}$ (say with $n \geqslant 2$ ) and a bounded, open 1 set $U$ as in Figure 1 .

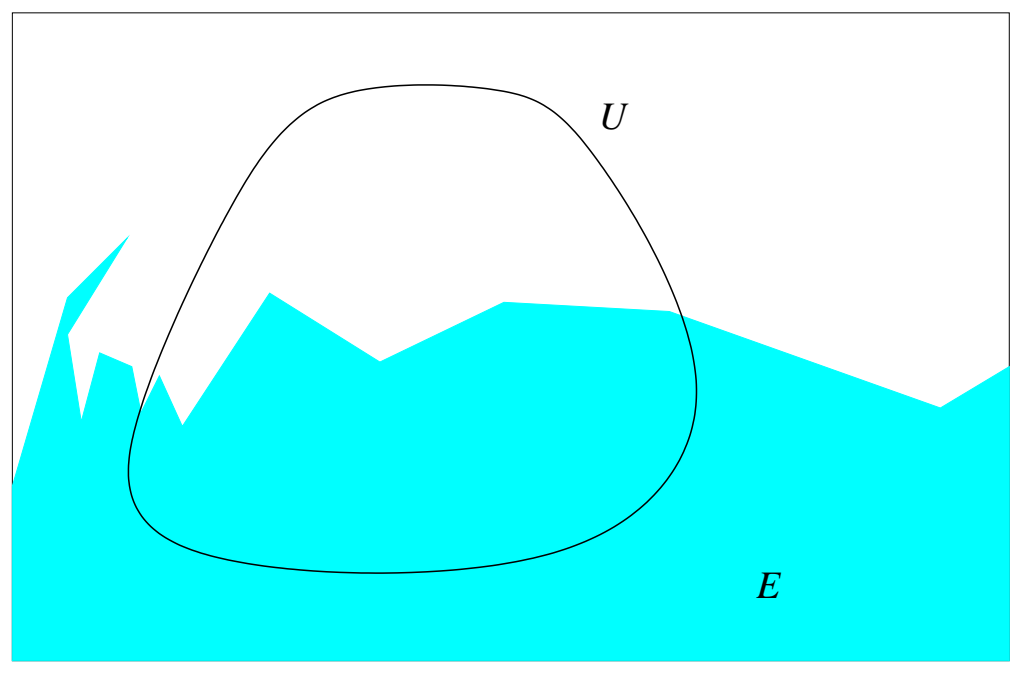

Figure 1. The sets $E$ (in gray) and $U$.

The main idea of the fractional perimeter is that any point inside $E$ "interacts" with any point outside $E$ giving rise to a functional whose minimisation is taken into account. On the other hand, in the functional one may neglect the interactions that are fixed as "boundary datum" since they cannot contribute to the minimisation (and they also may give an infinite contribution, which is safer to take away). That is, the set $O$ splits $E$ and its complement into four sets, two inside $E$, namely $E^{\prime}:=E \cap U$ and $E^{\prime \prime}:=E \backslash U$, and two outside $E$, namely $O^{\prime}:=U \backslash E$ and $O^{\prime \prime}:=\left(\mathbb{R}^{n} \backslash E\right) \cap\left(\mathbb{R}^{n} \backslash U\right)$, see Figure 2. Then the functional is the collection of the interactions of the points in $E^{\prime}$ and $E^{\prime \prime}$ with the points in $O^{\prime}$ and $O^{\prime \prime}$, with the exception of the interactions of points in $E^{\prime \prime}$ with the ones in $O^{\prime \prime}$, that are "fixed by the boundary values".

I am greatly indebted to Begoña Barrios, Luis Caffarelli, Serena Dipierro, Alessio Figalli, Giampiero Palatucci, Ovidiu Savin and Yannick Sire: the results outlined in this note are the fruit of the very pleasant and stimulating collaboration with them and I profited enormously from the possibility of having them as mentors and coworkers. This work is supported by the ERC project $\epsilon$ (Elliptic Pde's and Symmetry of Interfaces and Layers for Odd Nonlinearities) and the FIRB project A\&B (Analysis and Beyond).

${ }^{1}$ In the sequel, for simplicity, the domain $U$ will be often implicitly assumed connected and with smooth boundary. 


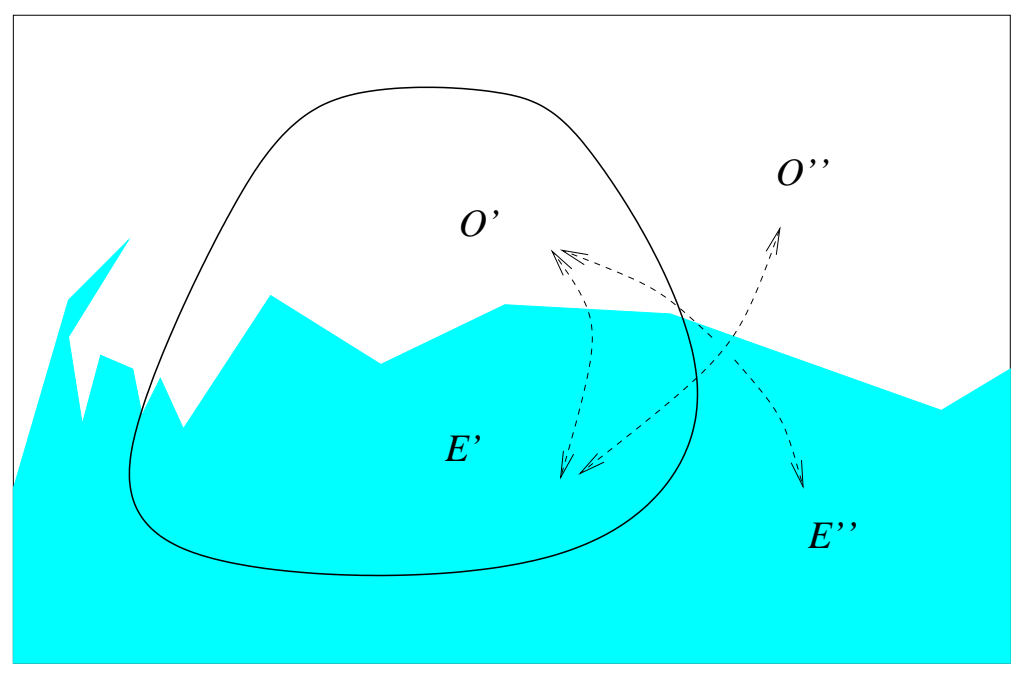

Figure 2. The sets $E^{\prime}, E^{\prime \prime}, O^{\prime}$ and $O^{\prime \prime}$ and their interactions.

Namely, one considers the functional

$$
\operatorname{Per}_{s}(E, U):=\mathscr{I}\left(E^{\prime}, O^{\prime}\right)+\mathscr{I}\left(E^{\prime}, O^{\prime \prime}\right)+\mathscr{I}\left(E^{\prime \prime}, O^{\prime}\right),
$$

which formally coincides with $\mathscr{I}\left(E, \mathbb{R}^{n} \backslash E\right)-\mathscr{I}\left(E^{\prime \prime}, O^{\prime \prime}\right)$ (though the latter may have no sense since both $\mathscr{I}\left(E, \mathbb{R}^{n} \backslash E\right)$ and $\mathscr{I}\left(E^{\prime \prime}, O^{\prime \prime}\right)$ could be infinite!). The interaction $\mathscr{I}$ that was considered in 8 ] is

$$
\mathscr{I}(A, B):=\iint_{A \times B} \frac{d x d y}{|x-y|^{n+2 s}}
$$

for any disjoint, measurable sets $A, B$ and for a fixed $s \in(0,1 / 2)$. The restriction on the range of $s$ is natural, since the integrals in (1.1) diverge in general when $s \in(-\infty, 0] \cup[1 / 2,+\infty)$ (more precisely, for $s \leqslant 0$ the contributions at infinity become unbounded, while for $s \geqslant 1 / 2$ the problem arises from point $x$ and $y$ arbitrarily close to each other). The functional in (1.1) naturally produces a minimisation problem: that is, one says that $E$ is $s$-minimal in $U$ if $\operatorname{Per}_{s}(E, U) \leqslant \operatorname{Per}_{s}(F, U)$ for any measurable set $F$ that coincides with $E$ outside $U$ (i.e., $F \backslash U=E \backslash U$ ).

The necessary compactness and semicontinuity properties to ensure the existence of such $s$ minimisers are proved in Section 3 of [8], and the following result was obtained:

Theorem 1.1 (Theorem 3.2 in [8]). Let $U \subset \mathbb{R}^{n}$ be a bounded Lipschitz domain and $E_{o} \subset \mathbb{R}^{n} \backslash U$ be a given set. There exists a set $E$, with $E \backslash U=E_{o}$ such that

$$
\operatorname{Per}_{s}(E, U) \leqslant \operatorname{Per}_{s}(F, U)
$$

for any $F$ such that $F \backslash U=E_{o} \backslash U$.

Moreover, in [8] $s$-minimisers are proved to satisfy a suitable integral equation, that is the EulerLagrange equation corresponding to the functional in (1.1). Namely suppose that $E$ is $s$-minimal 
in $U$ and that $x_{o} \in U \cap(\partial E)$ : then'2

$$
\int_{\mathbb{R}^{n}} \frac{\chi_{E}\left(x_{o}+y\right)-\chi_{\mathbb{R}^{n} \backslash E}\left(x_{o}+y\right)}{|y|^{n+2 s}} d y=0 .
$$

From the geometric point of view, (1.2) states that a suitable average of $E$ (centred at any point of $\partial E$ ) is balanced by the average of its complement. Due to the singularity of the denominator, (1.2) only makes sense for smooth sets: at this level, without knowing any a priori regularity for the set $E$, we must recall that equation (1.2) must be taken in the viscosity sense (we refer to Theorem 5.1 in [8] for details): in this setting, it may be interesting to notice that (1.2) says that $(-\Delta)^{s}\left(\chi_{E}-\chi_{\mathbb{R}^{n} \backslash E}\right)=0$ along $\partial E$ (see, e.g., [13] for a basic introduction on the fractional Laplacian operator).

Of course, the functional in (1.1) may present a cumbersome combinatorics which may complicate the computation of the interactions. One may somehow turn around this difficulty by reducing the minimisation problem in (1.1) to a pde problem in $\mathbb{R}^{n} \times(0,+\infty)$. For this, given $u: \mathbb{R}^{n} \rightarrow \mathbb{R}$, one introduces the extension of $u$ as

$$
\widetilde{u}(X):=\int_{\mathbb{R}^{n}} \mathscr{P}\left(x-y, x_{n+1}\right) u(y) d y \quad \text { with } \quad \mathscr{P}(X):=\frac{c_{n, s} x_{n+1}^{2 s}}{|X|^{n+2 s}} .
$$

Here we used the notation $X:=\left(x, x_{n+1}\right) \in \mathbb{R}^{n} \times(0,+\infty)$, and $c_{n, s}>0$ is a normalising constant. Given $\Omega \subset \mathbb{R}^{n+1}$ and $v: \mathbb{R}^{n} \times(0,+\infty) \rightarrow \mathbb{R}$, we define

$$
\mathscr{E}_{\Omega}(v):=\int_{\Omega \cap\left\{x_{n+1}>0\right\}} x_{n+1}^{1-2 s}|\nabla v(X)|^{2} d X .
$$

Then we have the following result:

Proposition 1.1 (Proposition 7.3 in [8]). The set $E$ is an s-minimiser in a ball $B$ if and only if the extension $\widetilde{u}$ of $\chi_{E}-\chi_{\mathbb{R}^{n} \backslash E}$ satisfies

$$
\mathscr{E}_{\Omega}(\widetilde{u}) \leqslant \mathscr{E}_{\Omega}(v)
$$

for all bounded Lipschitz domains $\Omega \subset \mathbb{R}^{n+1}$ with $\Omega \cap\left\{x_{n+1}=0\right\} \Subset B$ and all functions $v$ that equal $\widetilde{u}$ in a neighbourhood of $\partial \Omega$ and take the values \pm 1 on $\Omega \cap\left\{x_{n+1}=0\right\}$.

Notice that the restriction for $v$ to take values \pm 1 "on the trace" $\left\{x_{n+1}=0\right\}$ (that is, to "agree with a set" on $\mathbb{R}^{n}$ ) causes several analytical difficulties in the choice of the admissible perturbations of $\widetilde{u}$.

One of the main questions addressed in $[8]$ is the one of the regularity of the $s$-minimisers: that is, it is shown there that $s$-minimisers are smooth sets outside a singular set of $(n-2)$-Hausdorff dimension:

Theorem 1.2 (Main Theorem 2.4 in [8]). If $E$ is an s-minimiser in $B_{1}$, then $\partial E \cap B_{1 / 2}$ is, to the possible exception of a closed set $\Sigma$ of finite $(n-2)$-Hausdorff dimension, a $C^{1, \alpha}$-hypersurface around each of its points.

\footnotetext{
${ }^{2}$ We adopt the standard notation for the characteristic function of a set $E$, namely
}

$$
\chi_{E}(x)=\left\{\begin{array}{lc}
1 & \text { if } x \in E \\
0 & \text { otherwise }
\end{array}\right.
$$


Notice that one expects $\partial E$ to be "an $(n-1)$-dimensional object", so the statement that $\Sigma$ is "an $(n-2)$-dimensional object" states that $\Sigma$ is somehow negligible inside $\partial E$, hence $\partial E$ is smooth near "the majority of its points". On the other hand, Theorem 1.2 leaves many questions open: for instance, is there any singular set at all? are there any singularities if the dimension $n$ is small enough? what do the $s$-minimisers look like?

Some of these questions will be discussed in further detail in $\S 1.2-1.3$, As for the latter problem, it is quite embarrassing to admit that, at the moment, there is a real lack of explicit examples: indeed, the only explicit $s$-minimiser known is the half-plane (which is in fact a minimiser in any domain $U$ ): this was proved in Corollary 5.3 in [8] and the proof is based on a comparison principle (i.e., if an $s$-minimiser is contained in some strip outside $U$, then it is contained in the same strip inside $U$ too). Notice that this lack of explicit examples does not prevent $s$-minimisers from existing (recall Theorem 1.11). In any case, at the moment, no $s$-minimiser with a non-void singular set is known.

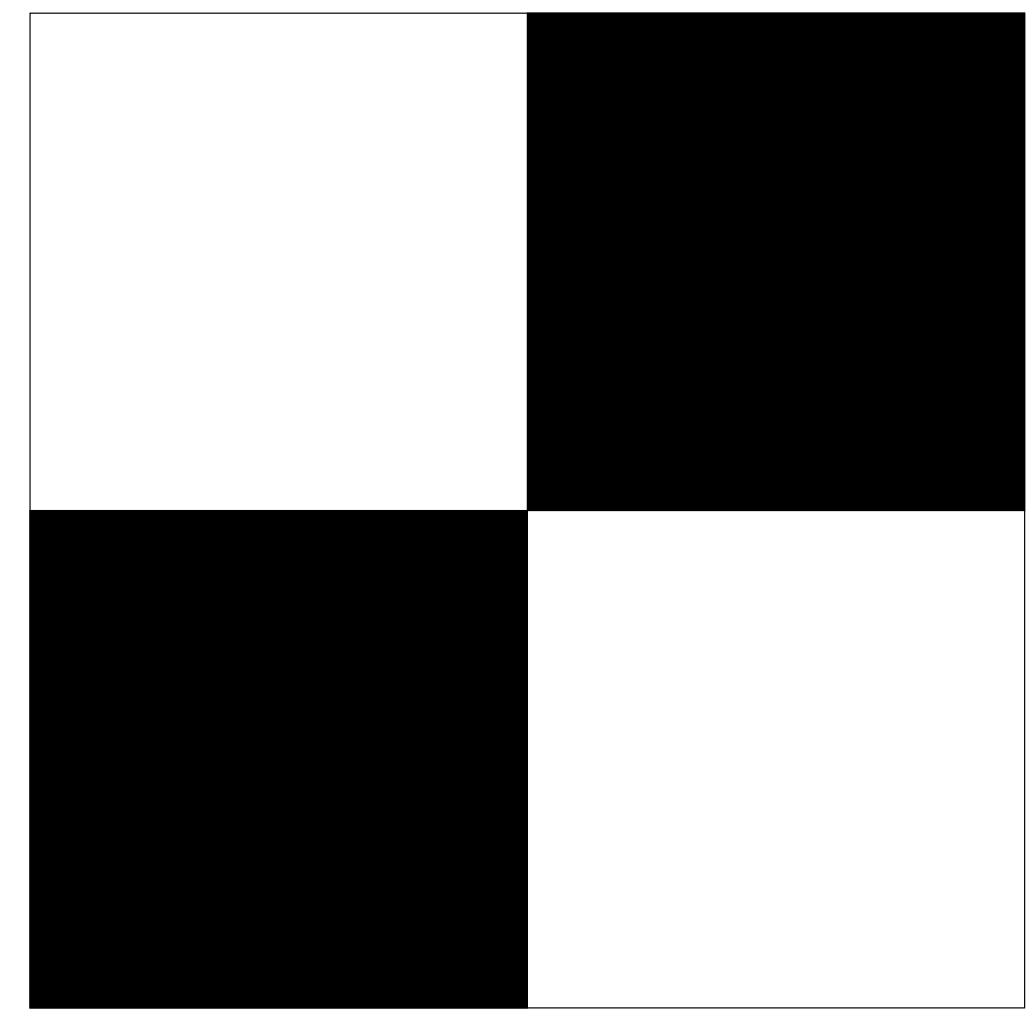

Figure 3. The cone $\mathscr{K}$.

One may also wonder if there are sets satisfying the Euler-Lagrange equation in (1.2) that possess a non-void singular set: the answer is in the affirmative, and a simple example is given by the classical cone in the plane

$$
\mathscr{K}:=\left\{(x, y) \in \mathbb{R}^{2} \text { s.t. } x y>0\right\},
$$

see Figure 3. Of course, $\mathscr{K}$ has a singularity at the origin, and, by symmetry, one sees that

$$
\mathscr{K} \text { satisfies (1.2) (possibly in the viscosity sense). }
$$

1.1. Asymptotics of the $s$-perimeter. Up to now, the reason for which we think that the functional in (1.1) is a "fractional perimeter" may seem mysterious to a reader not familiar with the subject. The motivation arises for the asymptotics as $s \nearrow 1 / 2$ in which the functional $\mathrm{Per}_{s}$ 
(suitably renormalised) approaches the classical perimeter (as usual, we use the notation $\omega_{n-1}:=$ $\mathscr{H}^{n-1}\left(S^{n-1}\right)$ for the surface of the $(n-1)$-dimensional sphere):

Theorem 1.3 ([9, 1]).

- Let $\alpha \in(0,1), R>0, s_{k} \nearrow 1 / 2$ and $E$ be a set with $C^{1, \alpha}$-boundary in $B_{R}$. Then

$$
\lim _{k \nearrow+\infty}\left(1-2 s_{k}\right) \operatorname{Per}_{s_{k}}\left(E, B_{r}\right)=\omega_{n-1} \operatorname{Per}\left(E, B_{r}\right) \quad \text { a.e. } r \in(0, R) .
$$

- Let $R>r>0, s_{k} \nearrow 1 / 2$ and $E_{k}$ be such that

$$
\sup _{k \in \mathbb{N}}\left(1-2 s_{k}\right) \operatorname{Per}_{s_{k}}\left(E_{k}, B_{R}\right)<+\infty .
$$

Then, up to subsequence, $\chi_{E_{k}}$ converges in $L^{1}\left(B_{r}\right)$ to $\chi_{E}$, for a suitable $E$ with finite perimeter in $B_{r}$.

- Let $R>r>0$. Let $s_{k} \nearrow 1 / 2$ and $E_{k}$ be $s_{k}$-minimisers in $B_{R}$, with $\chi_{E_{k}}$ converging in $L^{1}\left(B_{R}\right)$ to $\chi_{E}$. Then $E$ has minimal perimeter in $B_{r}$. Also, $E_{k}$ approach $E$ uniformly in $B_{r}$, meaning that for any $\epsilon>0$ there exists $k_{o}$ (possibly depending on $r$ and $\epsilon$ ) such that if $k \geqslant k_{o}$ then $E_{k} \cap B_{r}$ and $B_{r} \backslash E_{k}$ are contained, respectively, in an $\epsilon$-neighbourhood of $E$ and of $\mathbb{R}^{n} \backslash E$.

Also, one can show that the convergence of the functional in (1.1) to the classical perimeter as $s \nearrow 1 / 2$ holds in a suitable $\Gamma$-convergence sense: see [1]. In any case, we hope that this motivates the notation of fractional perimeter introduced in (1.1). On the other hand, when $s \searrow 0$, it is conceivable that the functional in (1.1) must approach, in some sense, the Lebesgue measure $\mathscr{L}^{n}$ (up to scaling). To see this, let us recall the notion of Gagliardo seminorm of a function $u$ :

$$
[u]_{G, s}:=\sqrt{\iint_{\mathbb{R}^{2 n}} \frac{|u(x)-u(y)|^{2}}{|x-y|^{n+2 s}} d x d y} .
$$

By taking the Fourier transform, one sees that

$$
[u]_{G, s}^{2}=c(n, s) \int_{\mathbb{R}^{n}}|\xi|^{2 s}|\widehat{u}(\xi)|^{2} d \xi,
$$

for any $u$ in the Schwartz space $C_{\downarrow}^{\infty}\left(\mathbb{R}^{n}\right)$ of rapidly decreasing smooth functions: here above $\widehat{u}$ is the Fourier transform of $u$ and $c(n, s)$ is a suitable normalising constant with the property that

$$
\lim _{s \searrow 0} c(n, s) s=c_{n}
$$

for an appropriate $c_{n}>0$ (see, e.g., Proposition 3.4 and Corollary 4.2 in [13]). Therefore

$$
\begin{aligned}
\lim _{s \searrow 0} s & {[u]_{G, s}^{2}=\lim _{s \searrow 0} c(n, s) s \int_{\mathbb{R}^{n}}|\xi|^{2 s}|\widehat{u}(\xi)|^{2} d \xi } \\
& =c_{n} \int_{\mathbb{R}^{n}}|\xi|^{0}|\widehat{u}(\xi)|^{2} d \xi=c_{n}\|\widehat{u}\|_{L^{2}\left(\mathbb{R}^{n}\right)}^{2}=c_{n}\|u\|_{L^{2}\left(\mathbb{R}^{n}\right)}^{2},
\end{aligned}
$$

thanks to Plancherel Theorem. Though this formula is obtained here for $u \in C_{\downarrow}^{\infty}\left(\mathbb{R}^{n}\right)$, it holds true also for functions $u \in L^{2}\left(\mathbb{R}^{n}\right)$ for which $[u]_{G, s_{o}}$ is finite for some $s_{o} \in(0,1)$ (see, e.g., [17] for a general theory in $L^{p}$-spaces). In particular, we may take $u:=\chi_{E}$ in (1.5) for a smooth $E \subset U$ 
(the smoothness of $E$ ensures that $\left[\chi_{E}\right]_{G, s_{o}}$ is finite and the boundedness of $E$ that $\chi_{E} \in L^{2}\left(\mathbb{R}^{n}\right)$ ): we conclude that

$$
\begin{gathered}
\lim _{s \searrow 0} 2 s \operatorname{Per}_{s}(E, U)=\lim _{s \searrow 0} 2 s \iint_{E \times\left(\mathbb{R}^{n} \backslash E\right)} \frac{d x d y}{|x-y|^{n+2 s}}=\lim _{s \searrow 0} s\left[\chi_{E}\right]_{G, s}^{2} \\
=c_{n}\left\|\chi_{E}\right\|_{L^{2}\left(\mathbb{R}^{n}\right)}^{2}=c_{n} \mathscr{L}^{n}(E) .
\end{gathered}
$$

The asymptotic behaviour as $s \searrow 0$ in the general case is slightly more complicated and it is dealt with in [14: the main difficulties are that the limit may not even exist and, when it does exist, it is a suitable convex combination of the normalised Lebesgue measure of $E \cap U$ with the one of $U \backslash E$, with the convex interpolation parameter depending on the "shape of $E$ outside $U$ ". More precisely, one introduces the parameter

$$
a(E):=\lim _{s \searrow 0} \frac{2 s}{\omega_{n-1}} \int_{E \backslash B_{1}} \frac{d y}{|y|^{n+2 s}}
$$

and the normalised Lebesgue measure $\mathscr{M}(E):=\omega_{n-1} \mathscr{L}(E)$. Notice that

$$
a(E) \leqslant \lim _{s \searrow 0} \frac{2 s}{\omega_{n-1}} \int_{\mathbb{R}^{n} \backslash B_{1}} \frac{d y}{|y|^{n+2 s}}=\lim _{s \searrow 0} 2 s \int_{1}^{+\infty} \frac{\rho^{n-1} d \rho}{\rho^{n+2 s}}=1
$$

hence $a(E) \in[0,1]$. Then, in some sense (that we will make precise below in Theorem 1.4), the asymptotic behaviour as $s \searrow 0$ is given by the formula

$$
\lim _{s \searrow 0} 2 s \operatorname{Per}_{s}(E, U)=(1-a(E)) \mathscr{M}(E \cap U)+a(E) \mathscr{M}(U \backslash E) .
$$

Notice that when $E$ is a smooth subset of $U$, then $E \subset B_{R}$ for some $R>0$, hence

$$
a(E) \leqslant \lim _{s \searrow 0} \frac{2 s}{\omega_{n-1}} \int_{B_{R} \backslash B_{1}} \frac{d y}{|y|^{n+2 s}}=\lim _{s \searrow 0} 2 s \int_{1}^{R} \frac{\rho^{n-1} d \rho}{\rho^{n+2 s}}=0,
$$

i.e. $a(E)=0$ and so (1.8) boils down to (1.6). Moreover, (1.8) states that the $s$-perimeter (suitably normalised, which has a non-local nature) approaches as $s \searrow 0$ the convex combinations of two measures "localised" in $U$ (namely $\mathscr{M}(E \cap U)$ and $\mathscr{M}(U \backslash E)$ ), but the combination parameter $a(E)$ takes into account the contribution of $E$ "coming from infinity". Though this is rather attractive, a rigorous statement has to take into account the possibilities that the above limits do not exist, and the precise result on the asymptotics as $s \searrow 0$ reads as follows:

Theorem 1.4 (Theorems 2.5 and 2.7 in 14]). Let $E$ be such that $\operatorname{Per}_{s_{o}}(E, U)<+\infty$ for some $s_{o} \in(0,1 / 2)$ and suppose that the limit defining a $(E)$ in (1.7) exists. Then the limit in (1.8) holds true.

Also, if $\operatorname{Per}_{s_{o}}(E, U)<+\infty$ for some $s_{o} \in(0,1 / 2)$ and $\mathscr{L}^{n}(E \cap U) \neq \mathscr{L}^{n}(U \backslash E)$, then the existence of the limit defining a $(E)$ in (1.7) is equivalent to (1.8).

The existence condition on the limit defining $a(E)$ in (1.7) cannot be removed from Theorem 1.4, since [14] also provides an example when such limit does not exist (and the limit in (1.8) does not exist as well). 


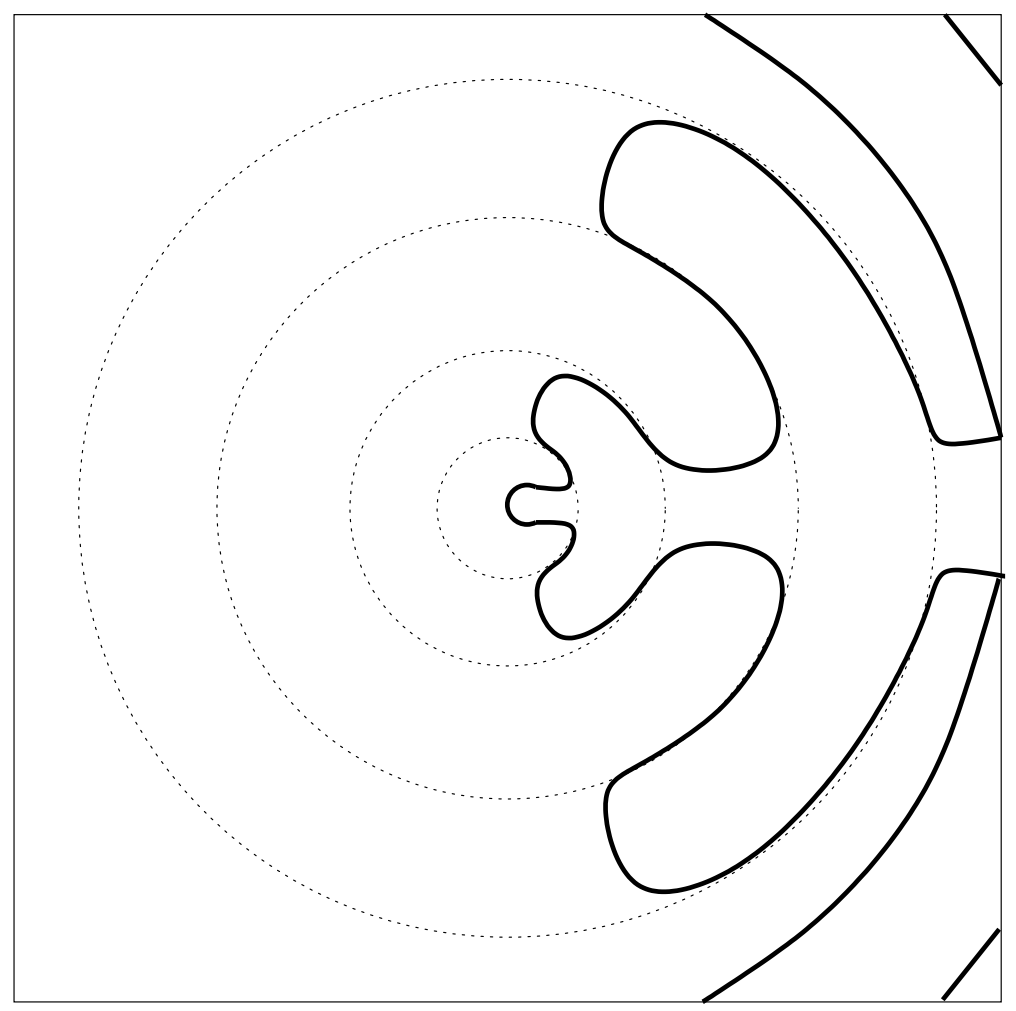

Figure 4. An example for which the limit as $s \searrow 0$ of the fractional perimeter does not exist.

Very roughly speaking, the example (as grossly depicted in Figure 4) considers a set $E$ which "looks like a cone" of a small opening in the annulus $B_{R_{2}} \backslash B_{R_{1}}$, then like a cone of a big opening in the annulus $B_{R_{3}} \backslash B_{R_{2}}$, and so on, alternating cones of small and big openings in subsequent annuli, with $R_{k} \rightarrow+\infty$ to be chosen appropriately. Then, the idea is that $a(E)$ would "feel alternatively" the small and the big cone openings in the asymptotics and consequently the limit in (1.7) does not exist (of course, some computation is needed to relate the "spatial oscillation" in the annuli with the parameter $s \searrow 0$, see Example 2.7 in [14 for details). Anyway, from Theorems 1.3 and 1.4, with a slight abuse of notation, one may think that the fractional perimeter interpolates the classical perimeter with a weighted Lebesgue measure when the parameter $s$ varies in the range $(0,1 / 2)$.

1.2. Regularity of $s$-minimal sets in the plane. Now we go back to the regularity issue of the $s$-minimal sets. Since this topic seems to be very difficult to deal with in the general case, we start with the case of low dimension $n=2$. For this, first we point out that

$$
\text { the cone } \mathscr{K} \text { in (1.3), that is the "black cone" in Figure 3, is not } s \text {-minimal. }
$$

The proof given here is due to an original idea of L. Caffarelli. Suppose, by contradiction, that $\mathscr{K}$ is $s$-minimal. Then consider the set $\mathscr{K}^{\prime}$ in Figure 5 that is obtained from $\mathscr{K}$ by adding another little square adjacent to the origin. 


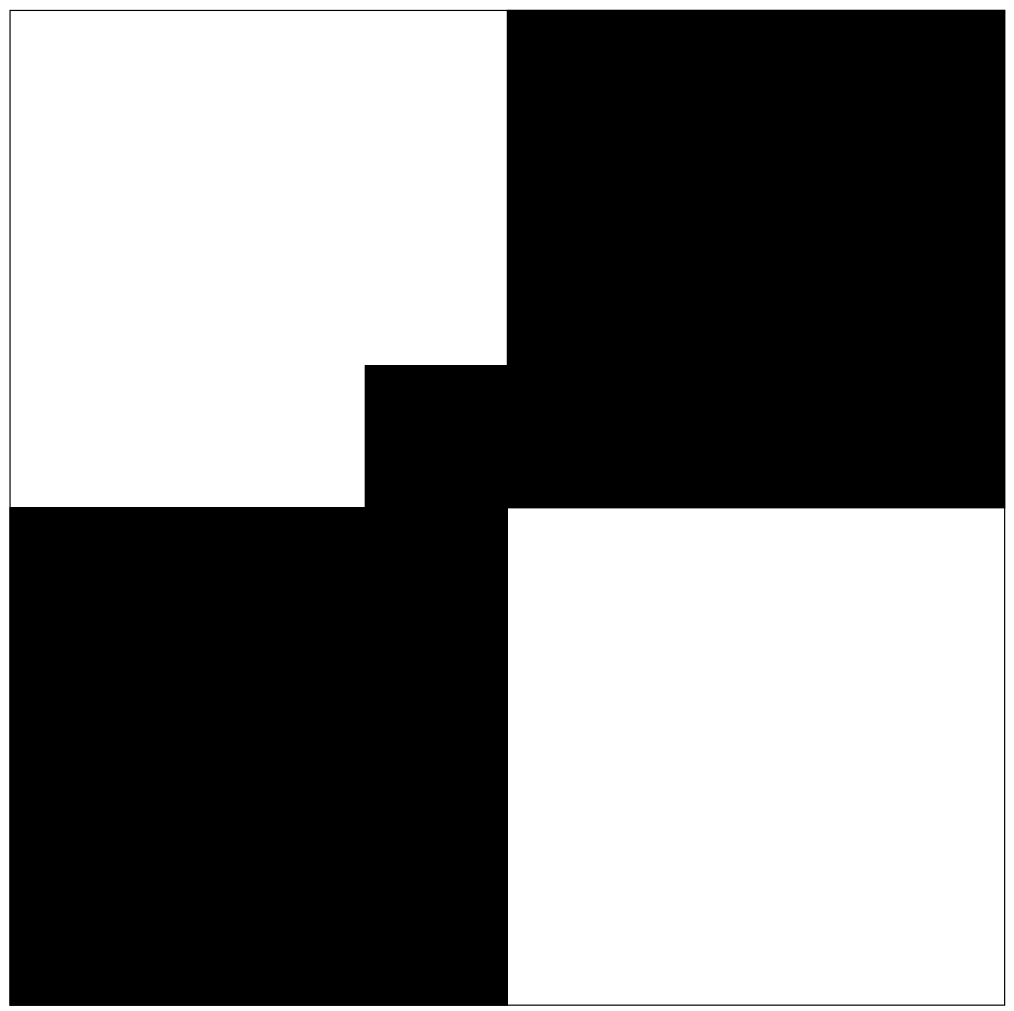

Figure 5. The cone $\mathscr{K}^{\prime}$.

Then, the $s$-perimeter of $\mathscr{K}^{\prime}$ (in a domain large enough to contain the additional little square) is equal to that of $\mathscr{K}$.

To check this, just compare Figures 6 and 7: in $\mathscr{K}$ the additional little square is "white" and therefore it interacts with the "black quadrants" $A$ and $B$ and with the "black rectangles" $C$ and $D$ in Figure 6, while in $\mathscr{K}^{\prime}$ the additional little square is "black" and therefore it interacts with the "white quadrants" $A^{\prime}$ and $B^{\prime}$ and with the "white rectangles" $C^{\prime}$ and $D^{\prime}$. Since the interactions with $A \cup B$ (resp., $C \cup D$ ) are equal to the ones with $A^{\prime} \cup B^{\prime}$ (resp., $C^{\prime} \cup D^{\prime}$ ), we have that the $s$-perimeter of $\mathscr{K}^{\prime}$ is equal to that of $\mathscr{K}$ (notice that - due to the finite space at our disposal Figures 6 and 7 only represent a "bounded portion" of $\mathbb{R}^{2}$, and the sets $A, B, C, D, A^{\prime}, B^{\prime}, C^{\prime}$ and $D^{\prime}$ are actually all unbounded). 


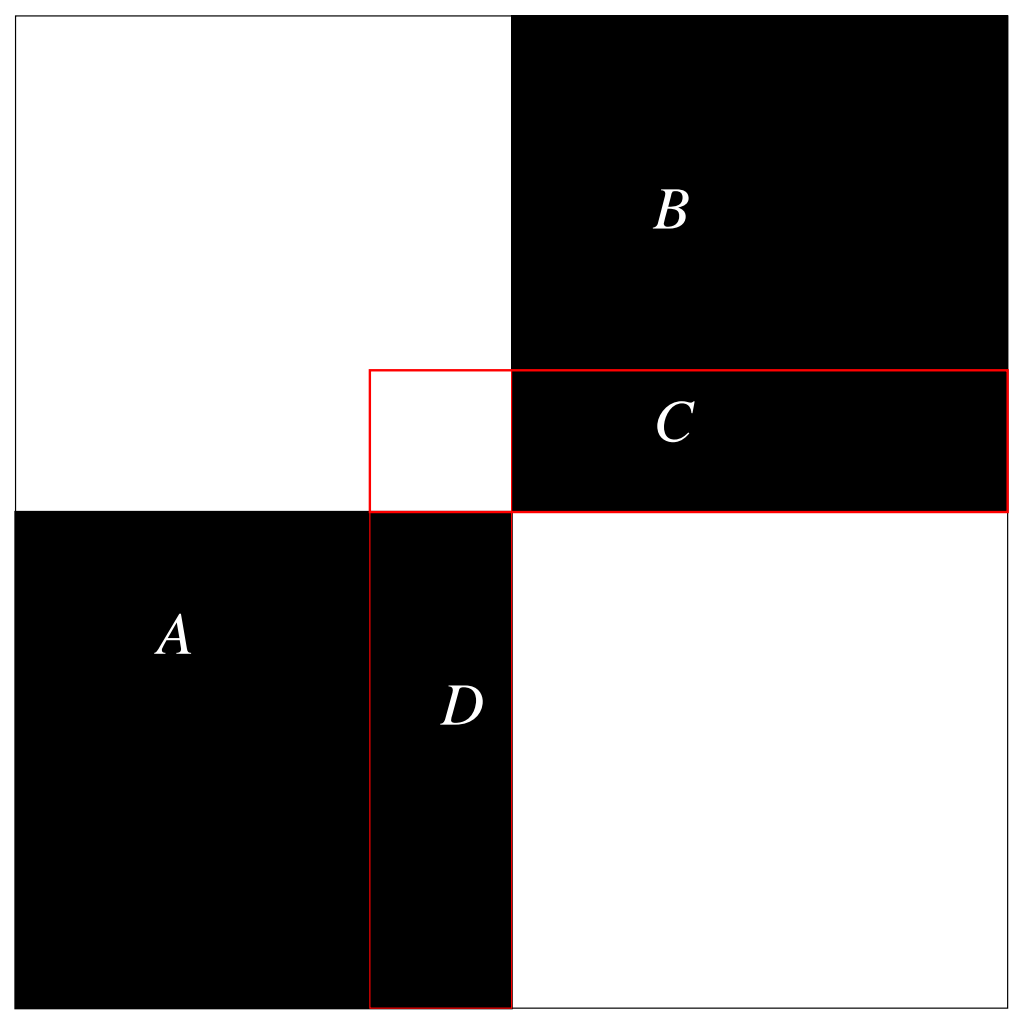

Figure 6. The sets $A, B, C$ and $D$ that interact with the little white square in $\mathscr{K}$.

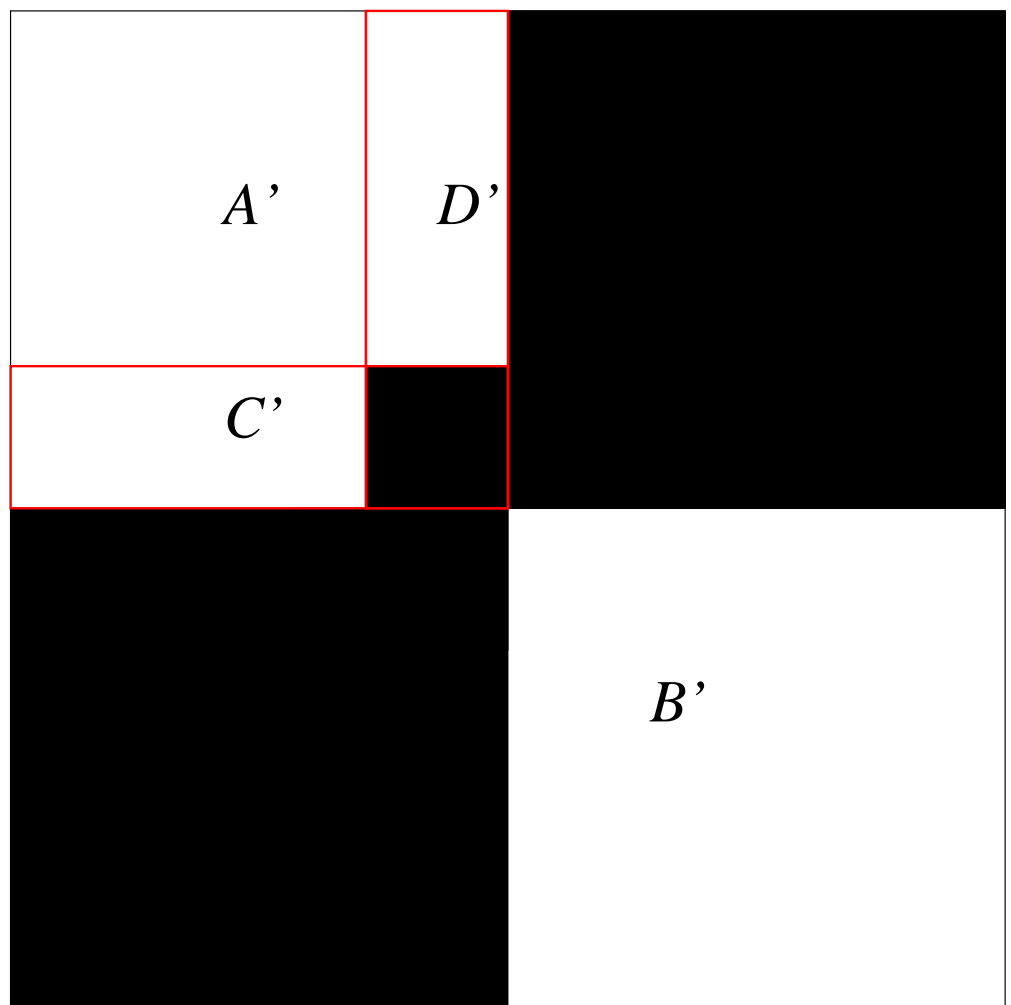

Figure 7. The sets $A^{\prime}, B^{\prime}, C^{\prime}$ and $D^{\prime}$ that interact with the little black square in $\mathscr{K}^{\prime}$. 
As a consequence, $\mathscr{K}^{\prime}$ is $s$-minimal (since we supposed that so is $\mathscr{K}$ ), and therefore $\mathscr{K}^{\prime}$ satisfies the Euler-Lagrange equation in (1.2) at the origin. But this cannot be, since the "black region" contributes more than the "white one", namely

$$
\int_{\mathbb{R}^{n}} \frac{\chi_{\mathscr{K}^{\prime}}(y)-\chi_{\mathbb{R}^{n} \backslash \mathscr{K}^{\prime}}(y)}{|y|^{n+2 s}} d y>0 .
$$

This contradiction shows that the cone $\mathscr{K}$ is not $s$-minimal, proving (1.9).

Moreover, recalling (1.4), we have that $\mathscr{K}$ is an example of a set that satisfies (1.2) but that is not $s$-minimal: thus the Euler-Lagrange equation in (1.2) is implied by, but it is not equivalent to, $s$-minimality.

It would be interesting to construct examples (if they exist) of smooth sets that satisfy the Euler-Lagrange equation in (1.2) without being $s$-minimal.

From (1.9) one may conjecture that $s$-minimal sets are smooth in dimension $n=2$ (I mean, if any singularity occurs, one can prove that one can reduce to a cone, and so one should suspect that the "worst" cone is the ninety degree one in Figure 3). Unfortunately it is not easy to extend the above geometric argument to the general case (for instance, the singular cone could be made of many sectors, and these sectors could differ one from the other, see Figure 10). To get around this difficulty, in 23] the regularity of $s$-minimal sets in dimension 2 is proved using an analytic argument based on domain perturbations. The result obtained 3 is the following:

Theorem $1.5\left([23)\right.$. Let $n=2$. If $R>r>0$ and $E$ is an $s$-minimal set in $B_{R}$, then $(\partial E) \cap B_{r}$ is a $C^{\infty}$-curve. If $E$ is an s-minimal set in $B_{\rho}$ for every $\rho>0$, then $\partial E$ is a straight line.

As a byproduct of Theorem 1.5 and of a dimensional reduction in [8, one also improves Theorem 1.2, obtaining that the singular set $\Sigma$ in $\mathbb{R}^{n}$ has finite $(n-3)$-Hausdorff dimension (instead of $n-2$ : and we do not know whether or not this is optimal, see Theorem [1.6 below).

The last claim in Theorem 1.5] somehow states that fractional geodesics in the plane are straight lines, as happens in the classical case. The proof of Theorem 1.5] is based on domain perturbation. The idea of the proof may be sketched by thinking about classical geodesics in the plane.

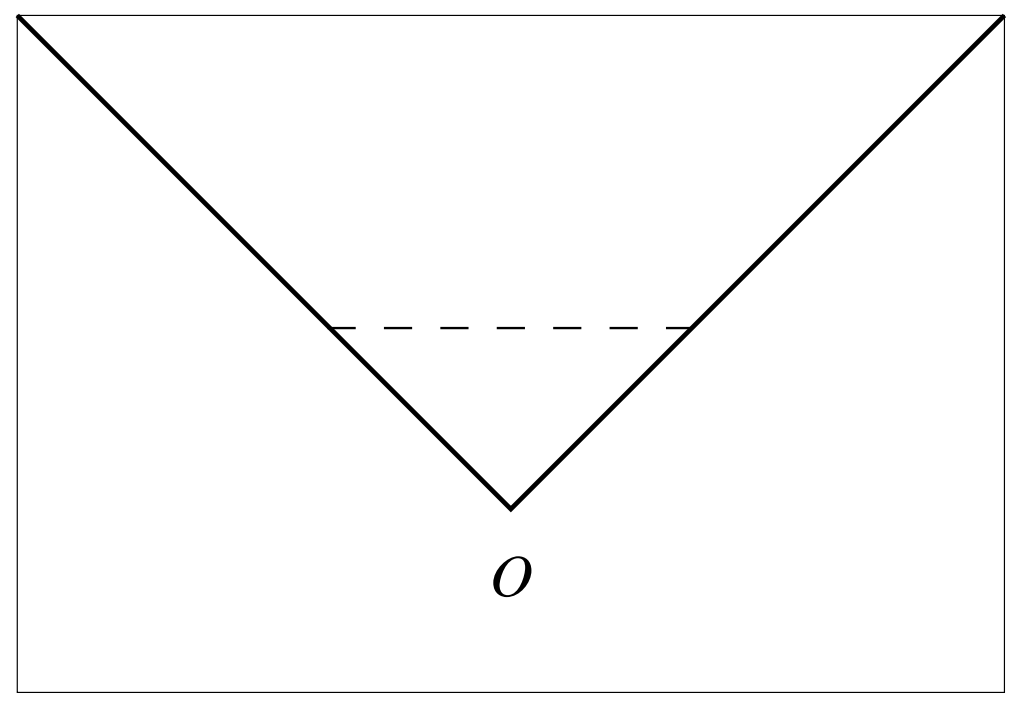

\footnotetext{
${ }^{3}$ In the regularity results such as Theorem 1.5 we are implicitly ruling out the trivial cases in which either $E$ or its complement is empty. Also, for the sake of precision, we point out that in 23 the regularity obtained is only of $C^{1, \alpha}$-type: the bootstrap improvement towards $C^{\infty}$-regularity is not trivial and it is contained in [2].
} 
Figure 8. An edge in the plane is not a geodesic: the classical proof

The classical proof to show that an edge is not a geodesic consists in cutting the angle in $O$ and shortening the length by the dashed segment as shown in Figure 8. This type of proof is difficult to transpose into a fractional framework, since the new object is not a smooth deformation of the original one. But there is a modification of this argument that shortens the length by taking a domain perturbation near $O$ of the edge plus a suitable gluing at infinity. This alternative argument is depicted in Figure 9.

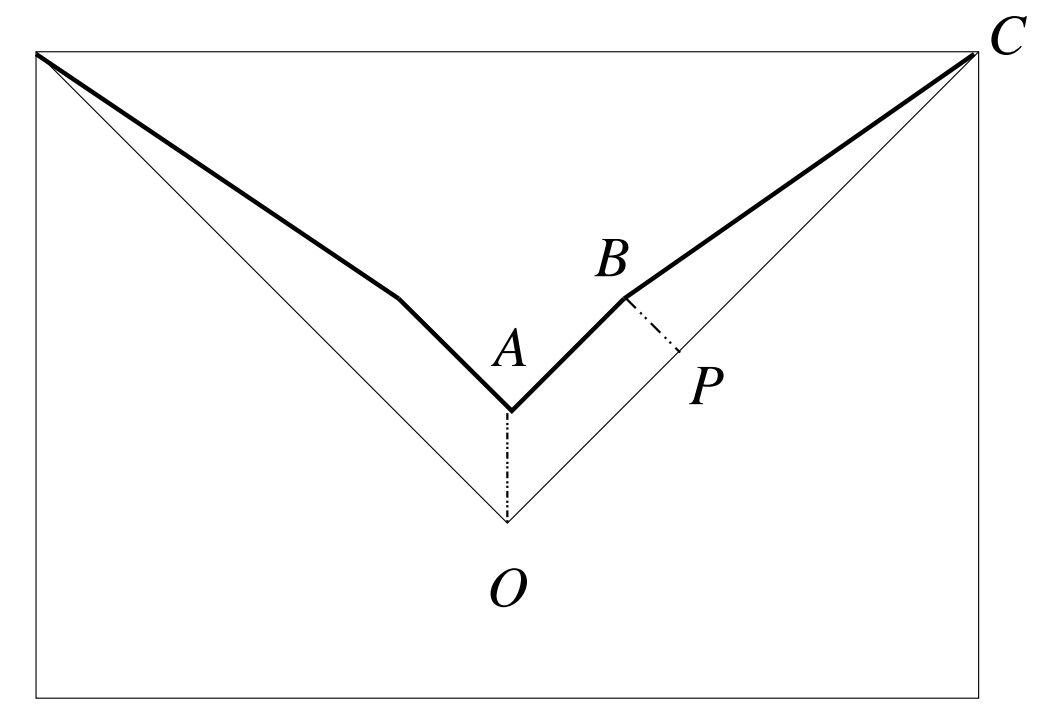

Figure 9. An edge in the plane is not a geodesic: the domain variation proof.

The argument goes like this (we follow the right side of the picture, the left one being symmetric). We translate the vertex slightly upwards, say, in such a way that $A O$ has length 1. Then, the length of $A B$ is strictly shorter than the one of $O P$, say

$$
\overline{A B}=\overline{O P}-\delta
$$

for some $\delta>0$. This is not a contradiction yet, since $A B$ is not a compact modification of $O P$, so, for our purposes, we need to glue $A B$ with $O P$. For this, we take a suitably large $R>0$, and we join $B$ to the point $C$, which is chosen in the half line from $O$ to $P$ in such a way that $P C$ has length $R$. Then, by Pythagoras' Theorem,

$$
\overline{B C}=\sqrt{\overline{B P}^{2}+\overline{P C}^{2}} \leqslant \sqrt{\overline{A O}^{2}+\overline{P C}^{2}}=\sqrt{1+R^{2}} \leqslant R+\frac{C}{R} .
$$

Now, if $R$ is chosen large enough, we obtain that the polygonal chain $A B C$ is shorter than the segment $O C$, namely:

$$
\overline{O C}-\overline{A B C}=\overline{O P}+\overline{P C}-(\overline{A B}+\overline{B C}) \geqslant \delta+R-\left(R+\frac{C}{R}\right)=\delta-\frac{C}{R}>0 .
$$

The argument on the left in Figure 9 is the same, and so we have shown that the edge has a longer length than the polygonal chain on the top of it.

This alternative argument proving that the edges in the plane are not geodesic may be extended to the fractional case. That is, one considers a minimal cone $E \subset \mathbb{R}^{2}$ (different than a hyperplane) and constructs a set $\widetilde{E}$ as a translation of $E$ in $B_{R / 2}$ which coincides with $E$ outside $B_{R}$. Then we use Proposition 1.1 to compute the difference between the energies of the extensions of $\widetilde{E}$ and $E$ : we obtain that this difference is bounded by $C / R^{2 s}$ (notice that formally the limit case $s=1 / 2$ 
goes back to the term $C / R$ in (1.11)). Technically, this estimate is achieved by considering a smooth perturbation $\phi \in C_{0}^{\infty}\left(\mathbb{R}^{3}\right)$, with $\phi(X)=1$ if $|X| \leqslant 1 / 2$ and $\phi(X)=0$ if $|X| \geqslant 3 / 4$, and considering the diffeomorphism

$$
\mathbb{R}^{3} \ni X \mapsto Y(X):=X+(\phi(X / R), 0,0) .
$$

The inverse diffeomorphism is denoted, with a slight but common abuse of notation, $\mathbb{R}^{3} \ni$ $Y \mapsto X(Y)=X$. Given $u$ to be the extension of $\chi_{E}$, according to Proposition 1.1, one defines $u_{R}^{+}(Y):=u(X)$. Similarly, by switching $\phi$ with $-\phi$ in (1.13), we can define $u_{R}^{-}(Y)$. Of course, the derivatives of $u^{ \pm}$may be computed from the ones of $u$ via the Chain Rule: in this way, we can compute $\mathscr{E}_{B_{R}}\left(u^{ \pm}\right)$and compare it with $\mathscr{E}_{B_{R}}(u)$ : one obtains

$$
\mathscr{E}_{B_{R}}\left(u_{R}^{+}\right)+\mathscr{E}_{B_{R}}\left(u_{R}^{-}\right)-2 \mathscr{E}_{B_{R}}(u) \leqslant \frac{C}{R^{2 s}} .
$$

Then, since $u$ is a minimiser, $\mathscr{E}_{B_{R}}(u) \leqslant \mathscr{E}_{B_{R}}\left(u_{R}^{-}\right)$and so we obtain

$$
\mathscr{E}_{B_{R}}\left(u_{R}^{+}\right)-\mathscr{E}_{B_{R}}(u) \leqslant \frac{C}{R^{2 s}} .
$$

Now we look at the cone $E$ in $\mathbb{R}^{2}$ : up to a rotation, we may suppose that a sector of $E$ has an angle less than $\pi$ and is bisected by $e_{2}$. Thus, there exist $M \geqslant 1$ and $p \in B_{M}$, on the $e_{2}$-axis, such that $p$ lies in the interior of $E$, and $p+e_{1}$ and $p-e_{1}$ lie in the exterior of $E$, and we let $P:=(p, 0) \in \mathbb{R}^{3}$ (see Figure 10 where $q:=p+e_{1}$ ).

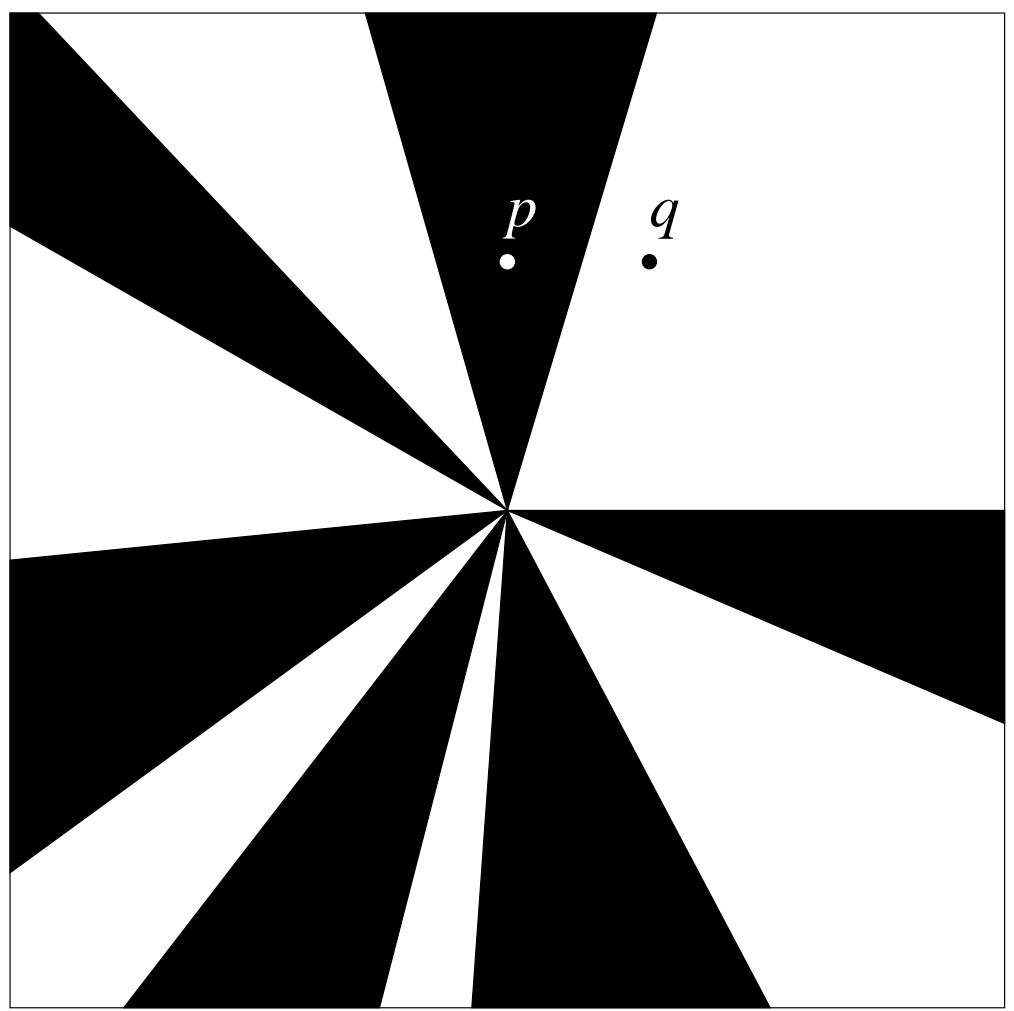

Figure 10. The singular cone $E$, with $p \in B_{M}$ and $q:=p+e_{1}$.

Taking $R$ much larger than $M$ we see that $u_{R}^{+}(Y)=u\left(Y-e_{1}\right)$ if $|Y| \leqslant 2 M$, and $u_{R}^{+}$coincides with $u$ if $|Y| \geqslant R$. We define

$$
v_{R}(X):=\min \left\{u(X), u_{R}^{+}(X)\right\} \quad \text { and } \quad w_{R}(X):=\max \left\{u(X), u_{R}^{+}(X)\right\} .
$$


By construction, $u_{R}^{+}<w_{R}=u$ in a neighbourhood of $P$, and $u<w_{R}=u_{R}^{+}$in a neighbourhood of $P+e_{1}$, that is $u$ and $u_{R}^{+}$cross each other inside the ball of radius $2 M$. This and the maximum principle imply that $w_{R}$ (as well as $v_{R}$ ) cannot be a minimiser with respect to compact perturbations in the ball of radius $2 M$ : that is, there exists $\delta>0$ and a modification $u_{*}$ of $w_{R}$ inside $B_{2 M}$ such that

$$
\mathscr{E}_{B_{2 M}}\left(u_{*}\right) \leqslant \mathscr{E}_{B_{2 M}}\left(w_{R}\right)-\delta
$$

Notice that this $\delta>0$ is independent of $R$ (since $w_{R}$ restricted to the ball of radius $2 M$ is simply the maximum between $u$ and its translation and so it is is independent of $R$ ). The role played by $\delta$ here is indeed analogous to the one of (1.10). Since $u_{*}=w_{R}$ outside $B_{2 M}$ we have that

$$
\mathscr{E}_{B_{R} \backslash B_{2 M}}\left(u_{*}\right)=\mathscr{E}_{B_{R} \backslash B_{2 M}}\left(w_{R}\right),
$$

and so (1.16) becomes

$$
\mathscr{E}_{B_{R}}\left(u_{*}\right) \leqslant \mathscr{E}_{B_{R}}\left(w_{R}\right)-\delta
$$

The advantage of working with (1.17) rather than (1.16) is that the energy domain is now the ball of radius $R$ (that is the domain that supports the perturbation), but $\delta$ is independent of $R$. The minimality of $u$ also gives that

$$
\mathscr{E}_{B_{R}}(u) \leqslant \mathscr{E}_{B_{R}}\left(v_{R}\right)
$$

Now, in light of (1.15), we point out the integral identity

$$
\mathscr{E}_{B_{R}}\left(v_{R}\right)+\mathscr{E}_{B_{R}}\left(w_{R}\right)=\mathscr{E}_{B_{R}}(u)+\mathscr{E}_{B_{R}}\left(u_{R}^{+}\right) .
$$

All in all, we have that

$$
\begin{array}{ll}
\mathscr{E}_{B_{R}}(u)-\mathscr{E}_{B_{R}}\left(u_{*}\right) \\
\text { by (1.17) } & \geqslant \mathscr{E}_{B_{R}}(u)-\mathscr{E}_{B_{R}}\left(w_{R}\right)+\delta \\
\text { by (1.19) } & =\mathscr{E}_{B_{R}}\left(v_{R}\right)-\mathscr{E}_{B_{R}}\left(u_{R}^{+}\right)+\delta \\
\text { by (1.18) } & \geqslant \mathscr{E}_{B_{R}}(u)-\mathscr{E}_{B_{R}}\left(u_{R}^{+}\right)+\delta \\
\text { by (1.14) } & \geqslant \delta-\frac{C}{R^{2 s}},
\end{array}
$$

which is strictly positive for $R$ large enough. This is in contradiction with the minimality of $u$ and so it completes the proof of Theorem 1.5.

Notice that (1.20) plays the role of (1.12) in this case. Furthermore, the technique used to prove Theorem [1.5 seems to work for a wide class of variational problems: see e.g. 24], where these ideas are exploited to prove monotonicity and symmetry results for minimisers and stable solutions of a very general class of functionals.

1.3. Regularity of $s$-minimal sets when $s$ is close to $1 / 2$. Having settled the regularity of $s$-minimal sets in the plane in Theorem [1.5, we discuss now the possible regularity in a higher dimension. As far as we know, this problem is open up to now. Though no example of a singular set in any dimension and for any $s \in(0,1 / 2)$ is known, the only regularity result available at the moment seems to be the following one, which recovers the classical minimal surface regularity theory when $s$ is sufficiently close $1 / 2$ :

Theorem 1.6 ([10]). For any $n \in \mathbb{N}$ there exists $\epsilon_{n} \in(0,1 / 2]$ such that if $s \in\left((1 / 2)-\epsilon_{n}, 1 / 2\right)$ then s-minimal sets are "as regular as the classical minimal surfaces in dimension n", namely:

- If $n \leqslant 7$ and $s \in\left((1 / 2)-\epsilon_{n}, 1 / 2\right)$, then any $s$-minimal set is locally a $C^{\infty}$-surface.

\footnotetext{
${ }^{4}$ As already pointed out in the footnote on page 10 in [10] the regularity theory is of $C^{1, \alpha}$-type: for the bootstrap to $C^{\infty}$-regularity see [2].
} 
- If $n=8$ and $s \in\left((1 / 2)-\epsilon_{8}, 1 / 2\right)$, then any $s$-minimal set is locally a $C^{\infty}$-surface except, at most, at countably many isolated points.

- If $n \geqslant 8$ and $s \in\left((1 / 2)-\epsilon_{n}, 1 / 2\right)$, then any s-minimal set is locally a $C^{\infty}$-surface outside a closed set $\Sigma \subset \partial E$ with finite $(n-8)$-Hausdorff dimension.

Of course, in the notation of Theorem 1.6, $\Sigma$ could well be empty. The finite $(n-8)$-Hausdorff dimension statement in Theorem 1.6 improves (when $s$ in the "right range") the previous ones mentioned in Theorem 1.2 and right below Theorem 1.5. Unfortunately the proof of Theorem 1.6 uses a compactness argument, therefore nothing is known on $\epsilon_{n}$ (except that it is a positive, universal quantity, depending only on $n$, but no explicit bound is available). Of course, from Theorem 1.5 we know that $\epsilon_{2}=1 / 2$ is fine for the regularity theory when $n=2$ (but this really comes from [23] and it cannot be proved with the argument in [10]). Of course, any explicit bound on $\epsilon_{n}$ would be welcome to make Theorem 1.6 applicable in concrete cases.

Notice also that, in view of Theorem 1.4, we know that for $s$ close to 0 the $s$-minimal sets seem related to the minimisers of the Lebesgue measure, for which no regularity result is possible (a set can have a small Lebesgue measure and possess a very wild boundary). Therefore, the regularity of $s$-minimal sets when $s$ is close to 0 (if it holds true) is conceivably more difficult than in the case in which $s$ is close to $1 / 2$.

\section{The fractional Allen-Cahn equation}

Classical minimal surfaces naturally arise in phase transition models. Similarly $s$-minimisers of the functional in (1.1) arise in non-local phase transition models, in which the classical diffusion term is replaced by the fractional Laplacian. To see this, let us briefly review the relation between phase transitions and minimal surfaces in the standard case. We take $W \in C^{2}(\mathbb{R})$ to be a "doublewell potential", say, for concreteness,

$$
W(t):=\frac{\left(1-t^{2}\right)^{2}}{4}
$$

Then, the classical Allen-Cahn (or scalar Ginzburg-Landau) phase coexistence model investigates the functional

$$
\mathscr{F}(u, U):=\int_{U} \frac{|\nabla u(x)|^{2}}{2}+W(u(x)) d x .
$$

The minimisers of this functional satisfy the Allen-Cahn equation

$$
-\Delta u=u-u^{3} \text { in } U \text {, }
$$

and they have a strong tendency to stay close to \pm 1 (which are the "pure phases" of the model) since these values kill the potential energy, while the gradient term forces the transition to occur with the least possible surface tension. These heuristic considerations can be made rigorous by introducing a parameter $\epsilon$ and by considering the rescaled minimiser

$$
u_{\epsilon}(x):=u(x / \epsilon) \text {. }
$$

Scaling $u$ to $u_{\epsilon}$ in (2.1) (and normalising by a multiplicative factor of order $\epsilon^{n-1}$ which does not change the notion of minimisers), one is lead to study the functional

$$
\mathscr{F}_{\epsilon}(u, U):=\int_{U} \frac{\epsilon|\nabla u(x)|^{2}}{2}+\frac{1}{\epsilon} W(u(x)) d x .
$$

Then, the following classical result holds true:

Theorem 2.1 ([18, 7]). 
- As $\epsilon \searrow 0, \mathscr{F} \epsilon \Gamma$-converges to the classical perimeter functional, i.e., for any set $E$ of locally finite perimeter, the following holds:

- For any $u_{\epsilon} \in L_{\mathrm{loc}}^{1}\left(\mathbb{R}^{n},[-1,1]\right)$ converging to $\chi_{E}-\chi_{\mathbb{R}^{n} \backslash E}$ in $L_{\mathrm{loc}}^{1}\left(\mathbb{R}^{n}\right)$, we have that

$$
\operatorname{Per}(E, U) \leqslant \liminf _{\epsilon \searrow 0} \mathscr{F}_{\epsilon}\left(u_{\epsilon}, U\right) ;
$$

- There exists $u_{\epsilon} \in L_{\mathrm{loc}}^{1}\left(\mathbb{R}^{n},[-1,1]\right)$ that converges to $\chi_{E}-\chi_{\mathbb{R}^{n} \backslash E}$ in $L_{\text {loc }}^{1}\left(\mathbb{R}^{n}\right)$ such that

$$
\operatorname{Per}(E, U) \geqslant \limsup _{\epsilon \searrow 0} \mathscr{F}_{\epsilon}\left(u_{\epsilon}, U\right) ;
$$

- The following compactness properties holds: if $u_{\epsilon} \in L_{\mathrm{loc}}^{1}\left(\mathbb{R}^{n},[-1,1]\right)$ and

$$
\sup _{\epsilon \in(0,1)} \mathscr{F}_{\epsilon}\left(u_{\epsilon}, U\right)<+\infty
$$

then there exists $E$ and a convergent subsequence such that $u_{\epsilon}$ converges to $\chi_{E}-\chi_{\mathbb{R}^{n} \backslash E}$ in $L_{\text {loc }}^{1}\left(\mathbb{R}^{n}\right)$.

- Fixed $R>r>0, \vartheta_{1}, \vartheta_{2} \in(-1,1)$, if $u_{\epsilon}$ minimises $\mathscr{F}_{\epsilon}$ in $B_{R}$ (i.e. if $\mathscr{F}\left(u, B_{R}\right) \leqslant$ $\mathscr{F}_{B_{R}}(u+\varphi)$ for any $\left.\varphi \in C_{0}^{\infty}\left(B_{R}\right)\right)$, and $u_{\epsilon}(0)>\vartheta_{1}$ then

$$
\mathscr{L}^{n}\left(B_{R} \cap\left\{u_{\epsilon}>\vartheta_{2}\right\}\right) \geqslant c R^{n},
$$

provided that $\epsilon<c\left(\vartheta_{1}, \vartheta_{2}\right) R$. Also, $\left\{u_{\epsilon}>\vartheta_{2}\right\}$ approaches $E$ uniformly in $B_{r}$, and $E$ minimises the perimeter in $B_{r}$ with respect to its boundary data.

The aim of the following pages is to present what happens to these results in the fractional framework and to discuss some possible consequences. For this, we first introduce a domain notation by setting

$$
\begin{aligned}
Q_{U} & :=(U \times U) \cup\left(\left(\mathbb{R}^{n} \backslash U\right) \times U\right) \cup\left(U \times\left(\mathbb{R}^{n} \backslash U\right)\right) \\
& =\mathbb{R}^{2 n} \backslash\left(\left(\mathbb{R}^{n} \backslash U\right) \times\left(\mathbb{R}^{n} \backslash U\right)\right) .
\end{aligned}
$$

The set $Q_{U}$ will represent the natural domain of a non-local interaction between particles in $\mathbb{R}^{n}$ : namely this interaction is represented by an integral over $\mathbb{R}^{n} \times \mathbb{R}^{n}$, but we will remove from this integral the contribution given by two particles both lying in the complement of $U$, since this will be considered fixed by the datum (this is the same type of renormalisation procedure performed in (1.1) ). More concretely, for any $s \in(0,1)$ we consider the functional

$$
\mathscr{G}(u ; U):=\iint_{Q_{U}} \frac{|u(x)-u(y)|^{2}}{2|x-y|^{n+2 s}} d x d y+\int_{U} W(u(x)) d x .
$$

Notice that the functional $\mathscr{G}$ differs from the functional $\mathscr{F}$ in (2.1) since the gradient part (i.e., the $H^{1}$-seminorm of $u$ in $U$ ) is replaced here by a double integral of Gagliardo type, which tries to mimic a long-range particle interaction energy. The Euler-Lagrange equation associated with $\mathscr{G}$ is

$$
(-\Delta)^{s} u=u-u^{3} \text { in } U
$$

which may be seen as a fractional variant of the classical Allen-Cahn equation in (2.2) (see, e.g., [16] for applications to fractional mean curvature flows). To obtain a functional on which a $\Gamma$ convergence problem is well-posed, we proceed as before, taking $u_{\epsilon}$ as in (2.3), and scaling $u$ to $u_{\epsilon}$ in (2.5): unlike the classical case, here it is also necessary to normalise by a multiplicative factor that depends on $s$, namely $\epsilon^{n-2 s}$ if $s \in(0,1 / 2), \epsilon^{n-1}$ if $s \in(1 / 2,1)$ and $\epsilon^{n-1} \log (1 / \epsilon)$ when $s=1 / 2$ (often, in fractional problems, a logarithmic correction at $s=1 / 2$ is necessary to 
match the case $s \in(0,1 / 2)$ with the case $s \in(1 / 2,1))$. This procedure leads to the following functional

$$
\mathscr{G}_{\epsilon}(u ; U):=\left\{\begin{array}{cc}
\iint_{Q_{U}} \frac{|u(x)-u(y)|^{2}}{2|x-y|^{n+2 s}} d x d y+\frac{1}{\epsilon^{2 s}} \int_{U} W(u(x)) d x, & \text { if } s \in(0,1 / 2), \\
\log (1 / \epsilon) \iint_{Q_{U}} \frac{|u(x)-u(y)|^{2}}{2|x-y|^{n+2 s}} d x d y+\frac{\log (1 / \epsilon)}{\epsilon} \int_{U} W(u(x)) d x, & \text { if } s=1 / 2, \\
\epsilon^{2 s-1} \iint_{Q_{U}} \frac{|u(x)-u(y)|^{2}}{2|x-y|^{n+2 s}} d x d y+\frac{1}{\epsilon} \int_{U} W(u(x)) d x, & \text { if } s \in(1 / 2,1) .
\end{array}\right.
$$

2.1. $\Gamma$-convergence and density estimates. In this non-local setting for phase transitions, one may recover the $\Gamma$-convergence and density estimates results of Theorem 2.1, though they now provide an alternative selection of local and non-local limit interfaces, according to the cases $s \in(0,1 / 2)$ and $s \in[1 / 2,1)$. Namely, the limit problem reduces to the $s$-perimeter functiona 5 when $s \in(0,1 / 2)$ and to the classical perimeter functional when $s \in[1 / 2,1)$. This is somehow consistent with the fact that the $s$-perimeter reduces to the classical perimeter as $s \nearrow 1 / 2$ (recall Theorem 1.3). Also, from the point of view of the applications, it suggests that the limit interfaces of the non-local Allen-Cahn phase transition may be either local or non-local according to whether the fractional parameter $s$ is above or below the critical threshold $1 / 2$ (that is, when $s \geqslant 1 / 2$ the nonlocal effect is lost by the limit interface). In further detail, the result obtained reads as follows 6

Theorem $2.2([20,21,22])$.

- As $\epsilon \searrow 0, \mathscr{G}_{\epsilon} \Gamma$-converges to the s-perimeter functional when $s \in(0,1 / 2)$ and the classical perimeter functional when $s \in[1 / 2,1)$

- The following compactness property holds: if $u_{\epsilon} \in L_{\mathrm{loc}}^{1}\left(\mathbb{R}^{n},[-1,1]\right)$ and

$$
\sup _{\epsilon \in(0,1)} \mathscr{G}_{\epsilon}\left(u_{\epsilon}, U\right)<+\infty
$$

then there exists $E$ and a convergent subsequence such that $u_{\epsilon}$ converges to $\chi_{E}-\chi_{\mathbb{R}^{n} \backslash E}$ in $L_{\text {loc }}^{1}\left(\mathbb{R}^{n}\right)$.

- Fixed $R>r>0, \vartheta_{1}, \vartheta_{2} \in(-1,1)$, if $u_{\epsilon}$ minimises $\mathscr{G}_{\epsilon}$ in $B_{R}$ and $u_{\epsilon}(0)>\vartheta_{1}$ then

$$
\mathscr{L}^{n}\left(B_{R} \cap\left\{u_{\epsilon}>\vartheta_{2}\right\}\right) \geqslant c R^{n},
$$

provided that $\epsilon<c\left(\vartheta_{1}, \vartheta_{2}\right) R$. Also, $\left\{u_{\epsilon}>\vartheta_{2}\right\}$ approaches $E$ uniformly in $B_{r}$, and $E$ minimises either the s-perimeter or the classical perimeter in $B_{r}$ with respect to its boundary data (depending on whether $s \in(0,1 / 2)$ or $s \in[1 / 2,1)$ ).

We try to translate the statement of Theorem 2.2 into an evocative picture that involves the parameters $\epsilon$ and $s \in(0,1)$. Namely, in Figure 11, $s$ ranges horizontally and $\epsilon$ vertically; on the top of the picture (corresponding to the case $\epsilon=1$ ) we have a phase transition function $u_{\epsilon}$ whose level sets as $\epsilon \searrow 0$ approach some $\partial E$, which is drawn in the bottom of the picture (which corresponds to the case $\epsilon=0)$. When $s \in(0,1 / 2)$ this $\partial E$ is an $s$-minimal set, while for $s \in[1 / 2,1)$ it is a

\footnotetext{
${ }^{5}$ The careful reader will have noticed that the $s$-perimeter functional is defined only for $s \in(0,1 / 2)$ while the fractional Allen-Cahn equation for any $s \in(0,1)$.

${ }^{6}$ Some preliminary work needed for the proof of Theorem 2.2 and a careful analysis of the one-dimensional case was also performed in 19 .
} 
classical minimal surface (since "classical minimal surfaces are nice" and "s-minimal sets might be somewhat wild" the picture is trying to distinguish between them by showing either smooth surfaces or singular cones).

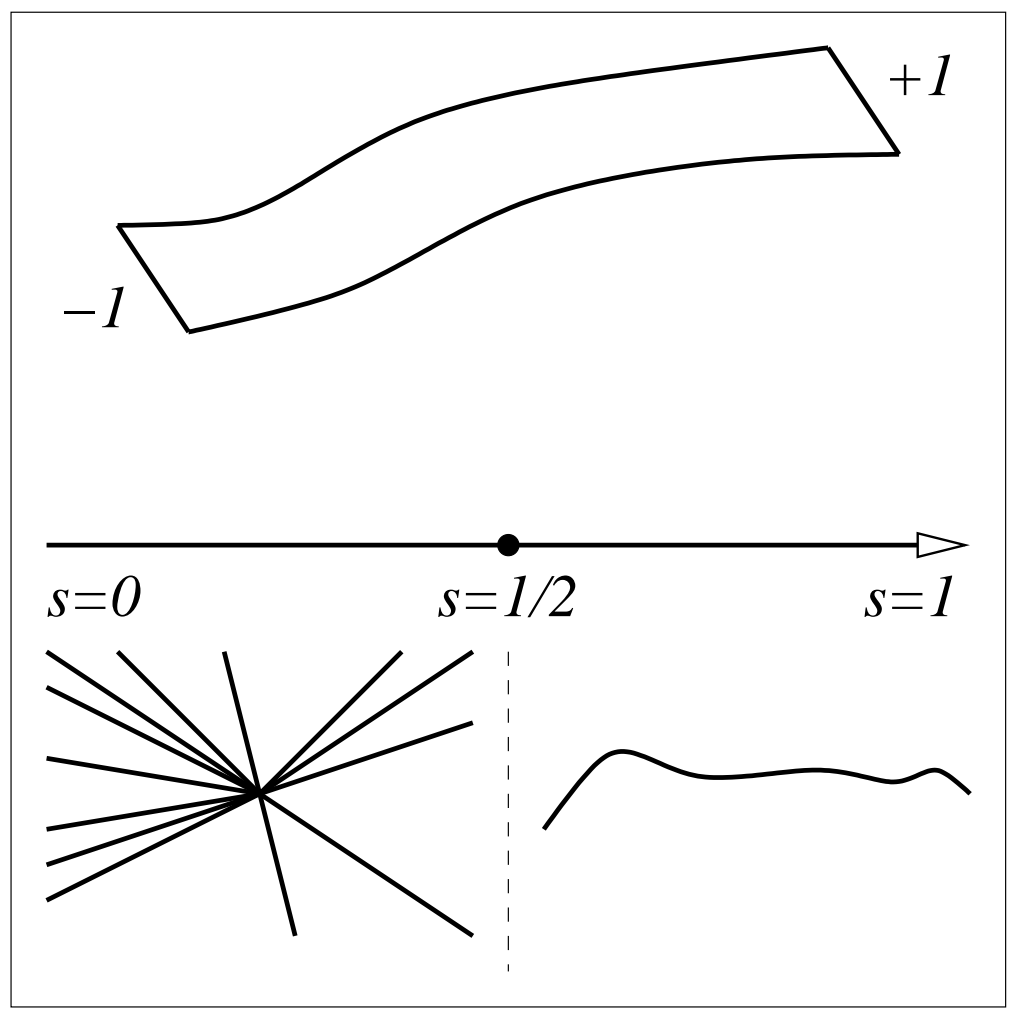

Figure 11. Г-convergence for fractional phase transitions.

As a matter of fact, the bottom of Figure 11 should be reconsidered in the light of Theorem 1.6: namely, at least when $n \leqslant 7$, the $s$-minimal sets should not look as "wild" as they were depicted, at least for $s \in\left((1 / 2)-\epsilon_{n}, 1 / 2\right)$ (and, when $n=2$, for any $s \in(0,1 / 2)$, recall Theorem 1.5) : we try to take into account this further regularity property in Figure 12, by extending the picture of the "nice" surface down to an unknown exponent $s=$. 


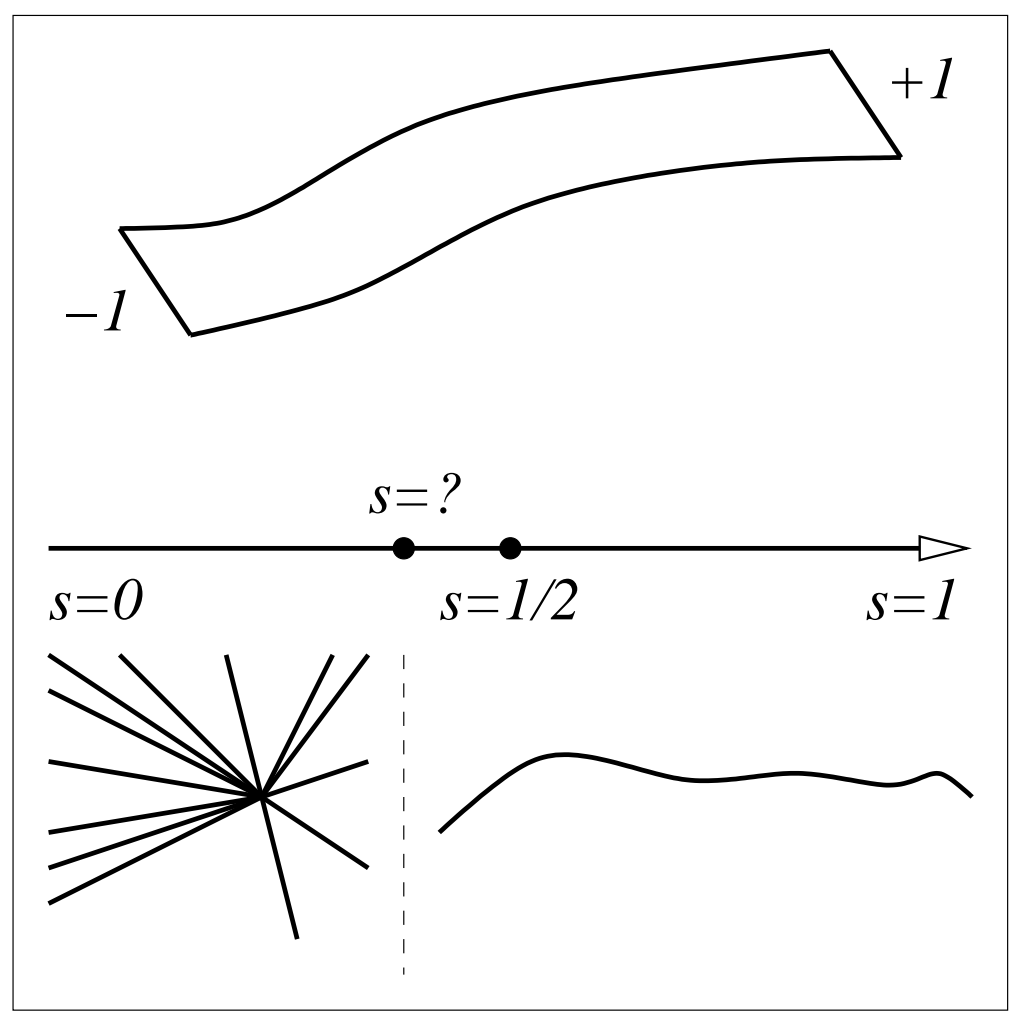

Figure 12. $\Gamma$-convergence for fractional phase transitions, taking into account Theorem 1.6 .

Now we discuss if and how these types of results may have an influence on the symmetry properties of the solution of the fractional Allen-Cahn equation.

2.2. One-dimensional symmetry. The classical Allen-Cahn equation (2.2) is linked to a very famous problem posed by De Giorgi:

Conjecture 2.1 ([1] $)$. Let $u \in C^{2}\left(\mathbb{R}^{n}\right) \cap L^{\infty}\left(\mathbb{R}^{n}\right)$ be a solution of the classical Allen-Cahn equation (2.2) in the whole of $\mathbb{R}^{n}$ and suppose that $\partial_{x_{n}} u(x)>0$ for all $x \in \mathbb{R}^{n}$.

Then, is it true that $u$ is one-dimensional (i.e., it depends only on one Euclidean variable up to rotation, and its level sets are hyperplanes), at least if $n \leqslant 8$ ?

Many outstanding mathematicians have given fundamental contributions to this problem and we cannot do justice here to all the results obtained and of all the important generalisations performed (see, e.g., [15] for a recent review on the topic): here we will just mention that Conjecture 2.1] is known to have an answer in the affirmative when $n \leqslant 3$ and in the negative when $n>8$. Also, it is conceivable that Conjecture 2.1 was inspired by the relation between the phase transitions and the minimal surfaces (recall Theorem 2.1) and by the rigidity and regularity features of the minimal surfaces.

Of course, a natural question is whether or not results inspired by Conjecture 2.1 hold true when the classical Allen-Cahn equation (2.2) is replaced by the fractional Allen-Cahn equation (2.6). This question was addressed in [6] when $n=2$ and $s=1 / 2$, in [25, 5] when $n=2$ and $s \in(0,1)$, in [3] when $n=3$ and $s=1 / 2$ and in [4] when $n=3$ and $s \in[1 / 2,1)$. We summarise these results in the following statement:

Theorem 2.3 ([ㄸ, 25, 5, 3, 4]). Let either

$$
n=2 \text { and } s \in(0,1)
$$


or

$$
n=3 \text { and } s \in[1 / 2,1) .
$$

Let $u \in C^{2}\left(\mathbb{R}^{n}\right) \cap L^{\infty}\left(\mathbb{R}^{n}\right)$ be a solution of the fractional Allen-Cahn equation (2.6) in the whole of $\mathbb{R}^{n}$ and suppose that $\partial_{x_{n}} u(x)>0$ for all $x \in \mathbb{R}^{n}$. Then $u$ is one-dimensional.

Clearly, Theorem 2.3 leaves many questions open. For instance, unlike the classical case, no counterexample is known in a higher dimension (it is conceivable, but not trivial to prove, that the counterexample in [12] works for $n>8$ and $s \in[1 / 2,1)$; the other ranges of $n$ and $s$ seem to be completely unknown). Furthermore, the ranges in (2.7) and (2.8) are still rather mysterious. One may think that these ranges are somehow reminiscent of the limit behaviour of the interfaces, according to Theorem 2.2. In this spirit, one may suspect that the symmetry result in Theorem 2.3 under condition (2.7) is a byproduct of the complete regularity theory of the minimisers of the related perimeter functionals (i.e., of the classical minimal surfaces when $s \in[1 / 2,1$ ), and of the $s$ minimal sets when $s \in(0,1 / 2)$, by Theorem 1.5). Similarly, one may suspect that the threshold $s=$ $1 / 2$ of condition (2.8) is an offspring of the same threshold that appears in Theorem 2.2, i.e. that the symmetry properties in Theorem 2.3 may break down in general when $s \in(0,1 / 2)$ due to the "wilderness" of the limit $s$-minimal sets. These arguments lead to the feeling that the threshold $s=1 / 2$ in (2.8) is optimal.

On the other hand, some other observations may lead to an opposite conclusion, that is the feeling that the threshold $s=1 / 2$ in (2.8) may be lowered a bit (maybe in dimensions $n=3, \ldots, 8$ ). Indeed, the proofs of Theorem 2.3 do not explicitly use any regularity properties of the (possibly fractional) minimal surfaces and the threshold $s=1 / 2$ in (2.8) does not come from geometric considerations but from analytical energy estimates. Moreover, if any relation between symmetry results for phase transitions and regularity results for (possibly fractional) minimal surfaces really holds true, then these regularity results for $s$-minimisers hold true up to a threshold $s=(1 / 2)-\epsilon_{n}$, so it seems conceivable that the symmetry properties may hold even slightly below $s=1 / 2$ at least when $n \leqslant 8$ (recall Theorem 1.6 and Figure 12). So we believe that any further investigation into the possible regularity of the $s$-minimal sets and the possible symmetries of the monotone solutions of the fractional Allen-Cahn equation would be a pleasant challenge for the researchers involved and a welcome progress for mathematics.

\section{REFERENCES}

[1] L. Ambrosio, G. De Philippis And L. Martinazzi, $\Gamma$-convergence of nonlocal perimeter functionals, Manuscripta Math. 134 (2011) 377-403.

[2] B. Barrios, A. Figalli AND E. VAldinoci, Bootstrap regularity for integro-differential operators and its application to nonlocal minimal surfaces, Ann. Sc. Norm. Super. Pisa Cl. Sci. (5) http://arxiv.org/abs/1202.4606v1

[3] X. Cabré, E. Cinti, Energy estimates and 1-D symmetry for nonlinear equations involving the half-Laplacian, Discrete Contin. Dyn. Syst. 28 (2010) (3) 1179-1206.

[4] X. Cabré, E. Cinti, Fractional diffusion equations: energy estimates and 1-D symmetry in dimension 3, preprint.

[5] X. Cabré, Y. SiRE, Nonlinear equations for fractional Laplacians II: existence, uniqueness, and qualitative properties of solutions, preprint arxiv.org/abs/1111.0796

[6] X. Cabré, J. SolÀ-Morales, Layer solutions in a half-space for boundary reactions, Comm. Pure Appl. Math. 58 (12) (2005) 1678-1732.

[7] L. Caffarelli And A. Cóndoba, Uniform convergence of a singular perturbation problem, Comm. Pure Appl. Math. 48 (1995) 1-12.

[8] L. Caffarelli, J.-M. Roquejoffre and O. Savin, Non-local minimal surfaces, Comm. Pure Appl. Math. 63 (2010) 1111-1144.

[9] L. Caffarelli And E. VAldinoci, Uniform estimates and limiting arguments for nonlocal minimal surfaces, Calc. Var. Partial Differential Equations 41 (1-2) (2011) 203-240. 
[10] L. Caffarelli and E. Valdinoci, Regularity properties of nonlocal minimal surfaces via limiting arguments, preprint http://arxiv.org/abs/1105.1158

[11] E. De GioRGI, Convergence problems for functionals and operators, Proceedings of the International Meeting on Recent Methods in Nonlinear Analysis, Bologna, Pitagora (1979).

[12] M. Del Pino, M. Kowalczyk And J. WeI, A counterexample to a conjecture by De Giorgi in large dimensions, C. R. Acad. Sci. Paris, Ser. I 346 (2008) 1261-1266.

[13] E. Di Nezza, G. Palatucci And E. Valdinoci, Hitchhiker's guide to the fractional Sobolev spaces, Bull. Sci. math. 136 (2012) 521-573.

[14] S. Dipierro, A. Figalli, G. Palatucci and E. Valdinoci, Asymptotics of the s-perimeter as $s \searrow 0$, Discrete Contin. Dyn. Syst. http://arxiv.org/abs/1204.0750

[15] A. Farina And E. VAlDinoci, The state of the art for a conjecture of De Giorgi and related problems, Recent progress on reaction-diffusion systems and viscosity solutions, World Sci. Publ., Hackensack, NJ (2009).

[16] C. IMBERT, Level set approach for fractional mean curvature flows, Interfaces Free Bound. 11 (2009) $153-176$.

[17] V. Mazya and T. Shaposhnikova, On the Bourgain, Brezis, and Mironescu theorem concerning limiting embeddings of fractional Sobolev spaces, J. Funct. Anal. 195 (2002) 230-238.

[18] L. ModicA, The gradient theory of phase transitions and the minimal interface criterion, Arch. Rational Mech. Anal. 98 (2) (1987) 123-142.

[19] G. PAlatucci, O. SAVIN AND E. VAldinOCI, Local and global minimizers for a variational energy involving a fractional norm, Ann. Mat. Pura Appl. (4), DOI: 10.1007/s10231-011-0243-9 http://dx.doi.org/10.1007/s10231-011-0243-9

[20] O. SAVIn AND E. VAldinOCI, Density estimates for a variational model driven by the Gagliardo norm, preprint http://arxiv.org/abs/1007.2114

[21] O. Savin And E. VAldinoci, Density estimates for a nonlocal variational model via the Sobolev inequality, SIAM J. Math. Analysis 43 (6) (2011) 2675-2687.

[22] O. Savin And E. Valdinoci, Г-convergence for nonlocal phase transitions, Ann. Inst. H. Poincaré Anal. Non Linaire 29 (4) (2012) 479-500.

[23] O. SAVin AND E. VAldinoci, Regularity of nonlocal minimal cones in dimension 2, Calc. Var. Partial Differential Equations, DOI: 10.1007/s00526-012-0539-7 http://www.springerlink.com/content/467n313161531332

[24] O. SAVIn AND E. VALDINOCI, Some monotonicity results for minimizers in the calculus of variations, preprint.

[25] Y. SiRE AND E. VALDINOCI, Fractional Laplacian phase transitions and boundary reactions: a geometric inequality and a symmetry result, J. Funct. Anal. 256 (6) (2009) 1842-1864.

Dipartimento di Matematica, Università degli Studi di Milano, Via Cesare Saldini 50, 20133 Milano (ITALY)

E-mail address: enrico.valdinoci@unimi.it 